\title{
Small Red Blood Cell Fraction on the UF-1000i Urine Analyzer as a Screening Tool to Detect Dysmorphic Red Blood Cells for Diagnosing Glomerulonephritis
}

Hyunjung Kim, M.D. ${ }^{1}$, Young Ok Kim, M.D. ${ }^{2}$, Yonggoo Kim, M.D. ${ }^{1}$, Jin-Soon Suh, M.D. ${ }^{3}$, Eun-Jung Cho, M.D. ${ }^{1}$, and Hae Kyung Lee ${ }^{10}$, M.D. ${ }^{1}$

Departments of ${ }^{1}$ Laboratory Medicine, ${ }^{2}$ Internal Medicine, and ${ }^{3}$ Pediatrics, College of Medicine, The Catholic University of Korea, Seoul, Korea

Background: Dysmorphic red blood cells (dRBCs) are first-line biomarkers for detecting glomerulonephritis (GN) in patients with hematuria. The UF-1000i system (Sysmex, Kobe, Japan), based on flow cytometry, provides small red blood cell (RBC) values (UF-1000i [UF]-\%sRBCs). We evaluated the clinical application of UF-\%sRBCs for detecting \%dRBCs and GN.

Methods: Urine samples of 103 patients (47 with GN; 56 without GN [NGN]) were analyzed using UF-1000i urinalysis, phase-contrast microscopy (PCM), and urine chemistry. Serum creatinine $(\mathrm{mg} / \mathrm{dL})$, serum albumin $(\mathrm{g} / \mathrm{dL})$, serum protein $(\mathrm{mg} / \mathrm{dL})$, urine protein $(\mathrm{mg} / \mathrm{dL})$, and urea nitrogen $(\mathrm{mg} / \mathrm{dL})$ levels were measured using an automated chemical analyzer. To determine the cut-off level of predicting GN, ROC curve was analyzed.

Results: UF-\%sRBCs, \%dRBCs, urine protein, serum creatinine, and estimated-glomerular filtration rate differed between the GN and NGN groups, with the greatest differences detected for UF-\%sRBCs and \%dRBCs $(P<0.0001)$. In ROC curve analysis, urine protein had the highest area under the curve (0.828), followed by \%dRBCs (0.771) and UF-\%sRBCs (0.745). To screen for GN, the best cut-off values of UF-\%sRBCs and \%dRBCs were $>40.5 \%$ and $>6.7 \%$, respectively. \%dRBCs $(P=0.0001)$ and UF-\%sRBCs $(P=0.0006)$ differed between the GN and NGN groups in patients with isolated hematuria but without proteinuria.

Conclusions: UF-\%sRBCs had similar diagnostic power to \%dRBCs determined by PCM for identifying patients with GN. UF-\%sRBCs may be more useful for diagnosing GN in patients with isolated hematuria. Predicting \%dRBCs using UF-1000i will provide information on possible GN in patients presenting with asymptomatic hematuria.

Key Words: Small red blood cell, Dysmorphic red blood cell, Hematuria, Glomerulonephritis, UF-1000i, Screening
Received: February 8, 2018

Revision received: June 28, 2018

Accepted: November 7, 2018

Corresponding author: Hae Kyung Lee, M.D. (1D https://orcid.org/0000-0002-9560-862X Department of Laboratory Medicine, Uijeongbu St. Mary's Hospital, College of Medicine, The Catholic University of Korea, 271 Cheonbo-ro, Uijeongbu 11765, Korea Tel: +82-31-820-3159

Fax: +82-31-847-6266

E-mail: hkl@catholic.ac.kr

\section{(c) Korean Society for Laboratory Medicine} This is an Open Access article distributed under the terms of the Creative Commons Attribution Non-Commercial License (http://creativecommons.org/licenses/by-nc/4.0) which permits unrestricted non-commercial use, distribution, and reproduction in any medium, provided the original work is properly cited.

\section{INTRODUCTION}

About $30-50 \%$ of patients diagnosed as having glomerulonephritis (GN) are first diagnosed as having an asymptomatic urinary abnormality [1]. Besides GN, there are various potential causes of hematuria, including tubular disease, urolithiasis, neoplasia, urinary tract or kidney infection, and rupture of the capillary vessels of the urinary system due to injury. The hematuria can then be classified as glomerular (the presence of dysmorphic red blood cells [dRBCs]) and non-glomerular (the pres- 
ence of isomorphic red blood cells [RBCs]) depending on the source of the bleeding [2, 3]. Microscopic examination of urine is routinely used to identify the morphology of RBCs in urine.

RBCs in GN show aberrant distributions, typically peaking at very small cell volumes, whereas RBCs in NGN show normal distributions, peaking in the normocytic to macrocytic range [4, 5]. Accordingly, the presence of dRBCs by microscopic examination is the first-line parameter used to detect GN in hematuria patients. When differentiating hematuria from GN and NGN, conventional microscopy techniques do not have good sensitivity and specificity for detecting dRBCs [6]. Therefore, to best identify dRBCs when distinguishing GN, phase-contrast microscopy (PCM) is the gold standard and is currently widely used. However, PCM is a time-consuming, labor-intensive, and insufficiently precise process that requires expert skills and knowledge, and is also influenced by the differences in sample preparation, such as amount of sample, centrifugation, and technician ability. Consequently, large inter-laboratory differences in the specificity and sensitivity of PCM have been reported [7, 8].

If glomerular hematuria and non-glomerular hematuria can be distinguished initially with an automated prompt analysis of micro-hematuria, urologic and nephrologic assessments can be performed easily and efficiently $[9,10]$. To rapidly and accurately treat and manage patients in this context, appropriate diagnostic methods should be used.

Although existing automated urine analyzers can detect RBCs and calculate the volume distribution curve, there is a risk of a spurious increase in the number of small RBCs due to misidentification of fragmented cells, crystals, microorganisms, debris, and urinary contaminants [11]. The UF-1000i automated urine particle analyzer (Sysmex, Kobe, Japan) uses fluorescence flow cytometry with semi-conductor laser technology for urinalysis [12]. Given that small RBCs are of smaller size but with extensive size variation $[13,14]$, we hypothesized that the UF-1000i small RBC (UF-\%sRBCs) parameter may predict the presence of dRBCs. We evaluated the clinical application of UF-\%sRBCs for detecting dRBCs and GN and analyzed the cutoff value of UF-\%sRBCs for diagnosing GN.

\section{METHODS}

\section{Study population and samples}

The present study was approved by the Institutional Review Board of the Catholic Medical Center (UC14SISI0172), and informed consent was obtained from all patients. A total of 103 patients (45 males, 58 females) were enrolled prospectively between
August 2015 and July 2016 at Uijeonbu St. Mary's Hospital, Uijeonbu, Korea. First, the patients with urine samples showing five or more RBCs per high-power field (HPF) on light microscopy were selected. We excluded patients with bacteriuria, urinary tract infection, or cystitis. The GN group comprised patients with pathologically confirmed GN by biopsy or overt proteinuria ( $>3,000 \mathrm{mg} /$ day on 24-hour urine collection). The NGN group comprised patients with microscopic hematuria from other urological abnormalities, such as kidney laceration and urolithiases, etc. Demographic and clinical data, including age, gender, baseline serum creatinine, comorbidities, and prior renal disease, were obtained from medical records.

Table 1 shows the results of \%dRBCs, UF-\%sRBCs, other UFRBC counts, and urine chemistry in each group. There were 47 patients with $\mathrm{GN}$, including IgA nephropathy $(\mathrm{N}=17)$, focal segmental glomerulosclerosis $(N=6)$, membranous nephropathy $(N=6)$, mesangial proliferative $G N(N=6)$, lupus nephritis $(N=6)$, and overt proteinuria $(\mathrm{N}=6)$. The NGN group included a total of 56 patients with kidney laceration $(\mathrm{N}=12)$, urolithiases $(\mathrm{N}=16$ [10 renal calculi, four ureteral calculi, and two bladder calculi]), bladder tumor ( $\mathrm{N}=11)$, and unspecified hematuria $(\mathrm{N}=17)$.

Urine was collected according to the CLSI recommendations [15]. Fresh urine samples with specific gravity of 1.003-1.030 were examined within 60-90 minutes of voiding. A midstream voided urine sample without preservatives was transferred to the laboratory immediately after collection. Urine samples were first analyzed using UF-1000i and then by PCM. All assays were performed as soon as possible and within one hours from the time of urine collection.

\section{Automated urinalysis}

UF-1000i uses fluorescence flow cytometry electrical impedance as an analytical principal and has two separate channels to stain urine sediments and bacteria [16]. In the flow cytometry analysis, when the light source hits the particles, the forward scatter channel (FSC) and the side scatter channel (SSC) generate the related values. The urine sample is then automatically mixed with reagents UF II PACK SED and UF II PACK B-A-C (Sysmex). Subsequently, 1,200 $\mu \mathrm{L}$ of the urine is aspirated and then split into two separate aliquots: one for bacteria counting and the other for analysis of other particles, such as RBCs, white blood cells, and casts. In general, bacteria or casts might falsely be recognized as RBCs, and this problem is more likely to occur for small RBCs. However, UF-1000i uses additional reagents (UF II SEARCH SED, UF II SEARCH B-A-C), which are based on the polymethine fluorescent dye. It has high sensitivity in de- 
Table 1. Demographic and laboratory parameters in patients with glomerulonephritis and non-glomerulonephritis

\begin{tabular}{|c|c|c|c|c|}
\hline Parameters* & $\begin{array}{l}\text { Glomerulonephritis } \\
\qquad(\mathrm{N}=47)\end{array}$ & $\begin{array}{l}\text { Non-glomerulonephritis } \\
\quad(\mathrm{N}=56)\end{array}$ & $\begin{array}{l}\text { Total patients } \\
(\mathrm{N}=103)\end{array}$ & $P$ \\
\hline Age (yr) & $54.1 \pm 17.8$ & $63.6 \pm 15.9$ & $58.9 \pm 17.0$ & 0.005 \\
\hline Male:Female & $21: 26$ & $24: 32$ & $45: 58$ & 0.853 \\
\hline UF total RBC count $(/ \mu \mathrm{L})$ & $1,450 \pm 4,284.8$ & $3,057 \pm 4,469.9$ & $2,137.70 \pm 4,187.1$ & 0.09 \\
\hline UF absolute small RBC (/ $/ \mathrm{L}$ ) & $48.4(21.5-201.4)$ & $104.3(25.6-286.5)$ & $67.5(25.6-246.7)$ & 0.32 \\
\hline UF-\%sRBC & $50.0 \pm 26.8$ & $23.7 \pm 26.9$ & $35.7 \pm 29.8$ & $<0.0001$ \\
\hline RBC (\%) & $33.3(6.5-58.5)$ & $3.0(1.0-9.5)$ & $6.2(1.0-41.2)$ & $<0.0001$ \\
\hline Urine protein (mg/dL) & $180.9(55.4-736.9)$ & $9.0(3.6-60.1)$ & $124.5(28.8--339.7)$ & 0.001 \\
\hline Blood urea nitrogen (mmol/L) & $6.9(5.1-10.1)$ & $6.0(4.4-9.1)$ & $6.8(4.9-9.4)$ & 0.46 \\
\hline Serum creatinine $(\mu \mathrm{mol} / \mathrm{L})$ & $113.2(78.7-158.2)$ & $87.6(66.3-105.2)$ & $97.2(70.7-141.4)$ & 0.004 \\
\hline eGFR (mg/dL/1.73 m²) & $55.9 \pm 29.8$ & $71.7 \pm 30.3$ & $67.1(44.7-91.5)$ & 0.01 \\
\hline Serum albumin (g/L) & $38.0 \pm 8.3$ & $35.7 \pm 7.2$ & $36.0 \pm 8.0$ & 0.89 \\
\hline Serum total protein (g/L) & $66.4 \pm 23.3$ & $66.6 \pm 8.8$ & $67.0 \pm 17.0$ & 0.96 \\
\hline
\end{tabular}

${ }^{*}$ Normally distributed variables are summarized as mean $\pm \mathrm{SD}$, and non-normally distributed variables are summarized as median (1st-3rd interquartile range). Abbreviations: UF, UF-1000i; BUN, blood urea nitrogen; eGFR, estimated-glomerular filtration rate; RBC, red blood cells; dRBC, dysmorphic RBCs.

tecting weak fluorescence and more accurately distinguishes bacteria, which are smaller than other particles and have low fluorescence intensities $[15,17]$. Therefore, the analysis of SSC light could minimize the false-positive RBC values derived from the casts. In addition to the usual urine parameters, UF-1000i provides information on research parameters, including the number of both small and large RBCs per microliter [18]. Small RBCs are counted as those with an FSC intensity value below 70. UF$\%$ sRBCs was calculated from the following formula: small RBC counts $(/ \mu \mathrm{L}) \times 100 /$ total RBC counts $(/ \mu \mathrm{L})$.

\section{PCM}

Urine samples ( $10 \mathrm{~mL}$ ) were centrifuged at $400 \times \mathrm{g}$ for five minutes, and the sediment was resuspended in $0.5 \mathrm{~mL}$ of supernatant urine. Aliquots of the suspension $(20 \mu \mathrm{L})$ were then analyzed and counted in a Fuchs-Rosenthal counting chamber. Ten HPFs $(\times 400)$ and at least 100 RBCs were screened for all patients. Microscopic examinations were performed by two qualified medical technologists who were unaware of the results of other urine test results. The means of the two results were used as the final data for analysis.

We considered doughnut-shaped RBCs, RBCs with vesicleshaped protrusions, target configuration, irregular cell contour membranous fragmentation, and fragmented RBCs as dRBCs, according to previous reports [14, 19]. \%dRBCs was calculated with the following formula: dRBC count $\times 100 / 100$ RBC counts. A \%dRBCs value $<20 \%$ is usually used as the criterion to rule out NGN in laboratory tests $[20,21]$.

\section{Serum and urine chemistry}

Serum was collected using BD Vacutainer SST (Beckton Dickinson, NJ, USA) and then analyzed within one hour. Serum creatinine $(\mu \mathrm{mol} / \mathrm{L})$, serum albumin $(\mathrm{g} / \mathrm{L})$, serum protein $(\mathrm{g} / \mathrm{L})$, urine protein $(g / L)$, and urea nitrogen $(\mathrm{mmol} / \mathrm{L})$ levels were measured using an automated chemical analyzer (Hitachi 7600-110, Hitachi Co., Tokyo, Japan). The estimated glomerular filtration rate (eGFR, $\mathrm{mL} / \mathrm{min} / 1.73 \mathrm{~m}^{2}$ ) was calculated using the Chronic Kidney Disease Epidemiology Collaboration (CKD-EPI) equation [20].

\section{Statistical analysis}

Continuous variables were summarized as mean $\pm S D$ if normally distributed or median with interquartile range if not, and categorical variables were summarized as frequencies. The F-test was used to test for equality of groups. An ANOVA or the Kruskal-Wallis test was used for comparisons among three or more groups. Scatter diagrams were plotted between \%dRBCs measured by PCM and UF-\%sRBCs. To determine the cut-off level of predicting GN, ROC curve analysis of the area under the curve (AUC) was performed using the GN and NGN groups for comparison. Sensitivity, specificity, positive predictive values (PPV), and negative predictive values (NPV) were calculated. All tests were two-sided. $P<0.05$ was considered statistically significant. Statistical analyses were performed using MedCalc 17.9.7 (MedCalc Software, Mariakerke, Belgium). 


\section{RESULTS}

UF-\%sRBCs and \%dRBCs values were correlated $(r=0.685$, $P<0.0001$; Fig. 1). When the patients were divided into positive and negative groups according to the $20 \%$ criterion, there were 34 patients with \%dRBCs >20\% and 33 patients with >20\% UF-\%sRBCs.

The laboratory parameters for the GN and NGN groups are summarized in Table 1 . The fraction of \%dRBCs and UF-\%sRBCs were higher in the GN group than in the NGN group (both $P<$ 0.001). UF-\%sRBCs urine protein, urea nitrogen, and serum creatinine levels were significantly higher, whereas eGFR was significantly lower in the GN group than in the NGN group. However, the RBC and small RBC counts from UF-1000i, serum

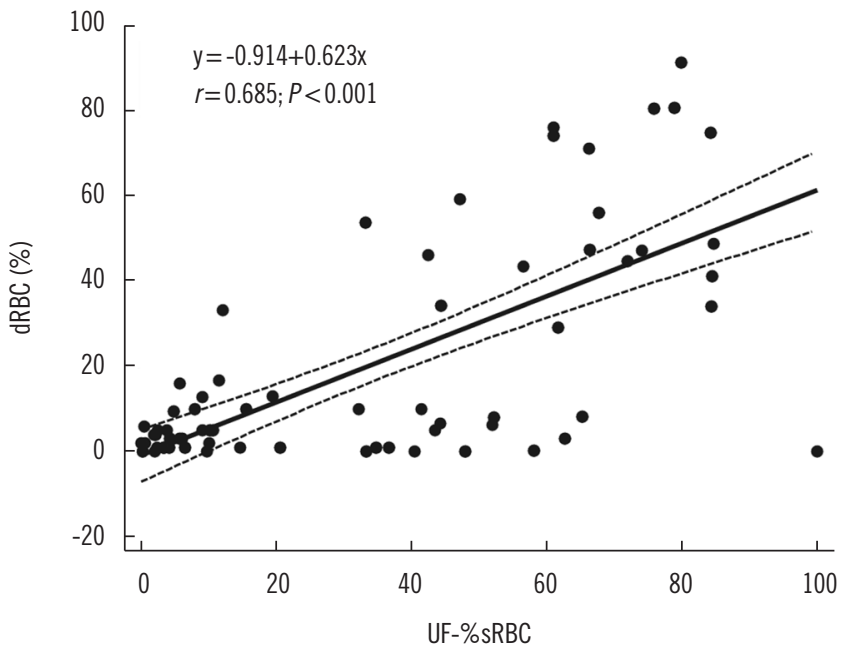

Fig. 1. Correlation between dysmorphic red blood cells (dRBCs) and UF-1000i small red blood cells (UF-\%sRBCs) $(r=0.685, P<0.0001)$. $\% \mathrm{dRBCs}$ was counted by phase-contrast microscopy. \%dRBCs $<20 \%$ was used as the criterion to rule out non-glomerular nephritis. urea nitrogen, serum albumin, and total protein did not differ significantly between the two groups. Since the total RBC count was higher in the NGN group than in the GN group, the absolute small RBC count was slightly higher in the NGN group. However, the absolute $\mathrm{dRBC}$ count is not an exact representation of RBC dysmorphia, which is why the $\mathrm{dRBC}$ "percentage" is commonly applied in the relevant literature. The absolute RBC and dRBC counts were higher in the NGN group than in the GN group, as reported previously, indicating that a higher \% $\mathrm{dRBC}$ is a more important marker of glomerular bleeding [21].

AUC and cut-off values of laboratory parameters for differentiating GN and NGN were analyzed from the ROC curve. The best cut-off of UF-\%sRBCs was $>40.5 \%$, with an AUC value of 0.745 , sensitivity of $70.2 \%$, and specificity of $76.8 \%$ (Table 2; Fig. 2). As shown in Table 2, urine protein was the parameter with the highest AUC value, followed by \%dRBCs and UF-\%sRBCs. The $\% \mathrm{dRBCs}$ and UF-\%sRBCs values showed similar sensitivity, specificity, PPV, and NPV (all $P<0.0001$ ). Overall, urine protein showed good sensitivity, PPV, and NPV, but relatively low specificity.

Next, to differentiate between patients with GN and NGN, we analyzed sensitivity and specificity based on the determined cutoff levels of \%dRBCs and UF-\%sRBCs. For UF-\%sRBCs, the cut-off value of $>14.6 \%$ had the best sensitivity, while $>44.4 \%$ showed optimum sensitivity and specificity. For \%dRBCs, the cut-off of $>6.7 \%$ had optimum sensitivity and specificity, while $>75 \%$ had the best specificity (Table 3 ).

We next focused on candidate parameters that significantly differ between the GN and NGN groups in the 53 patients with isolated hematuria (without proteinuria). As shown in Table 4, the \%dRBCs values were higher in the GN group with isolated hematuria than in the NGN group with isolated hematuria $(P=$

Table 2. Performance of laboratory parameters for the differentiation of glomerulonephritis and non-glomerulonephritis

\begin{tabular}{|c|c|c|c|c|c|c|c|}
\hline Parameters & AUC & Cut-off & Sensitivity (\%) & Specificity (\%) & PPV (\%) & NPV (\%) & $p^{*}$ \\
\hline dRBCs (\%) & $0.771(0.677-0.848)^{\dagger}$ & $>6.7$ & $74.5(59.7-86.1)^{\dagger}$ & $73.2(59.7-84.2)^{\dagger}$ & 70.0 & 77.4 & $<0.0001$ \\
\hline UF-\%sRBC & $0.745(0.650-0.826)$ & $>40.5$ & $70.2(55.1-82.7)$ & $76.8(63.6-87.0)$ & 70.2 & 75.0 & $<0.0001$ \\
\hline UF absolute small RBC (/ $/ L)$ & $0.558(0.456-0.655)$ & $>155.7$ & $72.3(57.4-84.4)$ & $44.6(31.3-58.5)$ & 48.0 & 100.0 & 0.31 \\
\hline UF total $R B C$ count $(/ \mu \mathrm{L})$ & $0.728(0.623-0.818)$ & $\leq 897.7$ & 91.7 (77.5-98.2) & 57.7 (43.2-71.3) & 34.2 & 47.7 & 0.0001 \\
\hline Urine protein (mg/dL) & $0.828(0.707-0.914)$ & $>9.8$ & $97.5(86.8-99.9)$ & $57.9(33.5-79.7)$ & 83.0 & 91.7 & $<0.0001$ \\
\hline Serum creatinine $(\mu \mathrm{mol} / \mathrm{L})$ & $0.669(0.565-0.761)$ & $>91.95$ & $57.5(42.2-71.7)$ & $79.6(65.7-89.8)$ & 69.2 & 64.9 & 0.002 \\
\hline $\mathrm{eGFR}\left(\mathrm{mg} / \mathrm{dL} / 1.73 \mathrm{~m}^{2}\right)$ & $0.664(0.560-0.757)$ & $\leq 65.9$ & $68.1(52.9-80.9)$ & $67.4(52.5-80.1)$ & 63.6 & 63.5 & 0.003 \\
\hline
\end{tabular}

${ }^{*} P$ values were obtained from ROC curve analysis for the differentiation of glomerulonephritis and non-glomerulonephritis; ${ }^{\dagger} 95 \%$ confidence intervals of AUC, sensitivity and specificity values.

Abbreviations: AUC, area under the curve; PPV, positive predictive value; NPV, negative predictive value; eGFR, estimated glomerular filtration rate; RBC, red blood cells; dRBC, dysmorphic RBCs; UF, UF-1000i. 
A

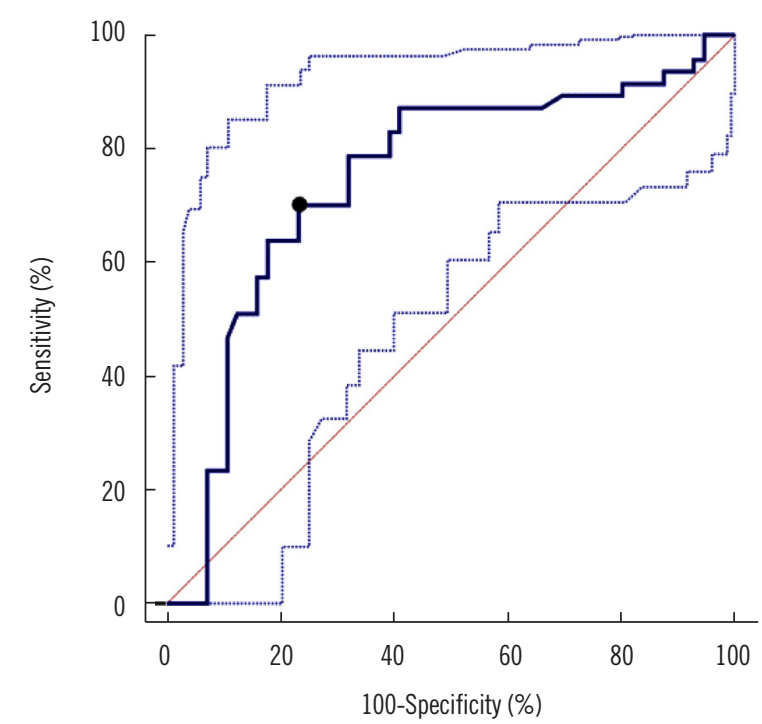

B

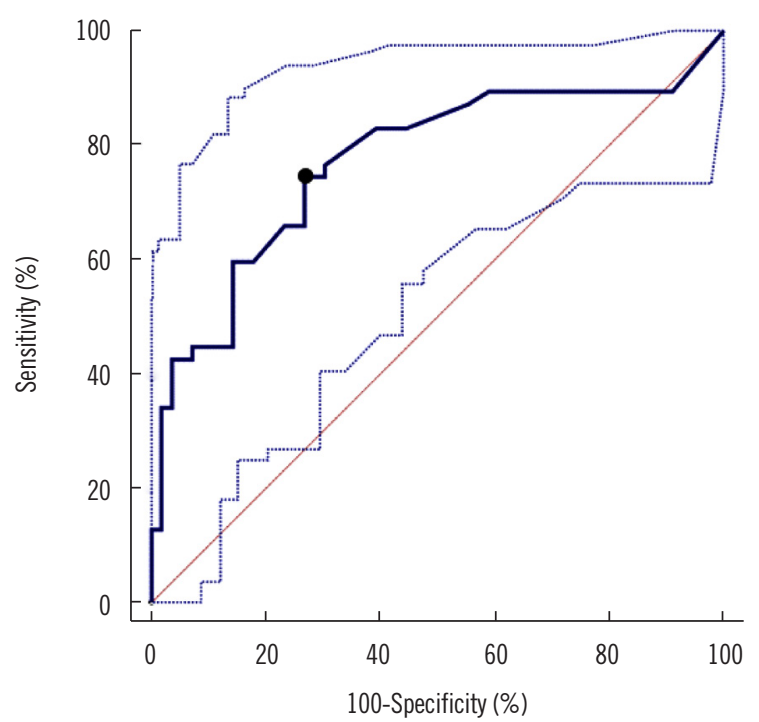

Fig. 2. ROC curves for UF-1000i small red blood cells (RBCs)\% (UF-\%sRBCs) and dysmorphic RBCs (\%dRBCs) to differentiate glomerulonephritis from non-glomerulonephritis patients. The cut-off was determined to be $(A)>40.5 \%$ with area under the curve $(A \cup C)=0.745$ for UF-\%sRBCs (B) $>6.7 \%$ with $A \cup C=0.771$ for \%dRBCs. Dotted lines are $95 \%$ confidence intervals of ROC cuves.

Table 3. Sensitivity and specificity values according to cut-off for UF-\%sRBCs (\%) and dRBC's (\%) for differentiating glomerulonephritis from non-glomerulonephritis

\begin{tabular}{lccccccc}
\hline \multirow{2}{*}{ Cut-off } & \multicolumn{3}{c}{ UF-\%sRBCs (\%) } & & \multicolumn{3}{c}{ dRBC (\%) } \\
\cline { 2 - 3 } & $>14.6$ & $>40.5$ & $>72.0$ & & $>6.7$ & $>10$ & $>75$ \\
\hline Sensitivity (\%) & $83.0(69.2-92.4)$ & $70.2(55.1-82.7)$ & $23.4(12.3-38.0)$ & & $74.5(59.7-86.1)$ & $59.6(44.3-73.6)$ & $12.8(4.8-25.7)$ \\
Specificity (\%) & $60.7(46.8-73.5)$ & $76.8(63.6-87.0)$ & $92.9(82.7-98.0)$ & & $73.2(59.7-84.2)$ & $82.1(69.6-91.1)$ & $98.2(90.4-100.0)$
\end{tabular}

Abbreviations: RBC, red blood cells; UF-\%sRBCs, UF Small RBC (\%); dRBC, dysmorphic RBC's; UF, UF-1000i.

Table 4. Major parameters between glomerulonephritis and nonglomerulonephritis groups in 53 patients with isolated hematuria (i.e., hematuria without proteinuria)

\begin{tabular}{lccc}
\hline Parameters* & $\begin{array}{c}\text { Glomerulonephritis } \\
\text { group }(\mathrm{N}=14)\end{array}$ & $\begin{array}{c}\text { Non- } \\
\text { glomerulonephritis } \\
\text { group (N=33) }\end{array}$ & $P$ \\
\hline dRBC (\%) & $47.3(16.10-56.20)$ & $13.10(1.00-5.00)$ & 0.0001 \\
UF-\%sRBC & $58.8(47.20-66.40)$ & $9.70(4.10-35.20)$ & 0.0006 \\
Serum creatinine $(\mu \mathrm{mol} / \mathrm{L})$ & $73.2(54.14-95.31)$ & $68.63(58.71-82.35)$ & 0.292 \\
eGFR (mg/dL/1.73 m²) & $75.1(44.80-105.00)$ & $81.40(71.60-96.50)$ & 0.477 \\
\hline
\end{tabular}

*The data are described as median (1st-3rd interquartile range). Abbreviations: eGFR, estimated-glomerular filtration rate; RBC, red blood cells; dRBC, dysmorphic RBCs; UF, UF-1000i.

0.0001). UF-\%sRBCs was also significantly higher in the GN group. When the patients with isolated hematuria were divided into positive and negative groups by the general criterion of the GN screening (dRBCs >20\%) [20, 21], nine of the $14 \mathrm{GN}$ patients with isolated hematuria (64.3\%) and five of the 33 NGN patients with isolated hematuria (15.2\%) had positive results for \%dRBCs. In addition, 11 of the 14 GN patients with isolated hematuria (78.6\%) and 17 of the 33 NGN patients with isolated hematuria (51.5\%) had positive results for UF-\% sRBCs. Serum creatinine and eGFR levels did not significantly differ between the two groups with isolated hematuria.

\section{DISCUSSION}

We evaluated the accuracy of automated detection of dRBCs and the diagnostic utility of UF-\%sRBCs between patients diagnosed as having GN and NGN, compared with the conventional PCM. The absolute RBC and $\mathrm{dRBC}$ counts were higher in the NGN group than in the GN group, as reported previously, indicating that a higher \%dRBC is a more important marker of glomerular bleeding [21].

Indications for renal biopsy vary among nephrologists but commonly include microhematuria with proteinuria, isolated protein- 
uria, overt proteinuria, chronic renal insufficiency, renal function deterioration, and evaluation of renal mass [22]. Although confirmation of isolated hematuria without proteinuria is a weak indication for renal biopsy, the identification of an increased dRBC count may guide the suspicion of GN and determine whether or not a renal biopsy should be performed [22, 23]. A renal biopsy is recommended in cases of isolated hematuria with a $\mathrm{dRBC}$ count $>80 \%$, RBC cast $>1$, acanthocytes $>5 \%$, three or more gross hematuria episodes, or hematuria associated with hypertension [24, 25]. \%dRBCs has emerged as a useful parameter for screening GN patients and could also be valuable to determine whether a renal biopsy should be performed when managing patients with hematuria without proteinuria. Our results further suggest the best cut-off value of UF-\%sRBCs for differentiating between GN and NGN patients.

The first choice of an imaging test for hematuria is a computed tomography (CT) urogram, which can detect the etiology of hematuria in approximately $41 \%$ of cases [26]. However, flexible cystoscopy, urine cytology, and CT urogram cannot be performed without a microscopic urinalysis confirming the presence of blood in the urine. Many patients with hematuria are subjected to unnecessary invasive procedures, including biopsy. In the present study, the urine protein level showed the best diagnostic power, followed by \%dRBCs and UF-\%sRBCs. Therefore, the use of a rapid and automatic parameter could help reduce the number of unnecessary renal biopsy or invasive tests as well as workloads and laboratory costs. Although \%dRBCs detection by PCM is an inexpensive hematuria assessment method, it has high inter-laboratory bias and requires skilled technicians and labor.

UF-\%sRBCs shows a diagnostic power similar to that of PCM for detecting GN patients. Most of patients (97.1\%) with \%dRBCs $>20 \%$ also showed a result of UF-\%sRBCs $>20 \%$. Therefore, the use of UF-\%sRBCs as a parameter has a low false-negative rate in finding \%dRBCs, thus having sufficient diagnostic power for a screening test. The parameters that significantly differed between the GN and NGN patients were UF-\%sRBCs, \%dRBCs, urine protein, serum creatinine, and eGFR. \%dRBCs and UF$\%$ sRBCs were moderately correlated $(r=0.685, P<0.0001)$, and UF-\%sRBCs accounted for most of dRBCs. The similar diagnostic power of UF-\%sRBCs and \%dRBCs may be related to the principle of UF-1000i.

Proteinuria quantification was found to be the parameter with the highest AUC to distinguish GN from NGN, followed by \%dRBCs and UF-\%sRBCs. Both proteinuria and hematuria can appear as initial symptoms in GN patients, and clinicians can easily decide onthe next evaluation steps in such cases. Crop et al. [27] reported that the combination of \%dRBCs and proteinuria provided the highest predicted probability for GN in ROC curve analysis. However, for patients with isolated hematuria without proteinuria, performing kidney biopsy is problematic. In the present study, $29.7 \%$ (14/47) of the patients with GN presented isolated hematuria without proteinuria, while $58.9 \%$ (33/56) of those with NGN had isolated hematuria. Therefore, \%dRBCs is a more valuable parameters to identify GN in patients with isolated hematuria as the initial symptom. Indeed, \%dRBCs and UF-\%sRBCs showed significant differences between patients with isolated hematuria in the GN and NGN groups; therefore, these markers can assist clinicians in deciding on the next evaluation step.

The best cut-offs for UF-\%sRBCs in differentiating GN from NGN were determined to be $>44.4 \%$ with the best sensitivity and specificity, whereas a \%dRBCs cut-off value $>75 \%$ showed low sensitivity and very high specificity; overall, the best cut-off was $>6.7 \%$ with $70.2 \%$ sensitivity and $70.2 \%$ specificity. This may be due to the strict counting of dRBCs and the inclusion of patients who were under treatment. In previous studies, \%dRBCs $>80 \%$ was used as the main criterion to indicate GN [13, 19, 28]. However, similar to our results, in one study adopting strict criteria [13], such as only counting acanthocyturia, \%dRBCs $>5 \%$ was suggested as the threshold, although other authors have regarded $>10 \%$ dRBCs to be indicative of GN $[19,28]$.

In conclusion, conventional automated urinalysis analyzers have difficulty in accurately determining \%dRBCs; however, UF$\%$ sRBCs is counted according to a principle combining impedance and flow cytometry. Therefore, the risk of misidentifying bacteria or casts is reduced, specifically when counting small RBCs. UF-\%sRBCs is an automation parameter that does not require substantial technical expertise for readings, thereby reducing labor and preventing variation. We demonstrated that UF-\%sRBCs has similar diagnostic power to \%dRBCs in identifying patients with GN, and may be particularly useful for patients with isolated hematuria. Patients with both hematuria and proteinuria have strong indications for renal biopsy [22]. Thus, in cases of isolated hematuria without proteinuria, clinicians may obtain rapid information regarding the possibility of GN by predicting \%dRBCs using UF-1000i.

\section{Authors' Disclosures of Potential Conflicts of Interest}

No potential conflicts of interest relevant to this article were reported. 


\section{Acknowledgment}

The authors thank Hyung-Jin Jeon for assistance with this research.

\section{REFERENCES}

1. Chae DW. Current status of primary glomerulonephritis. Korean. J Med 2013;84:1-5.

2. Fairley KF and Birch DF. Hematuria: a simple method for identifying glomerular bleeding. Kidney Int 1982;21:105-8.

3. De Santo NG, Nuzzi F, Capodicasa G, Lama G, Caputo G, Rosati P, et al. Phase contrast microscopy of the urine sediment for the diagnosis of glomerular and nonglomerular bleeding-data in children and adults with normal creatinine clearance. Nephron 1987;45:35-9.

4. Shichiri M, Hosoda K, Nishio Y, Ogura M, Suenaga M, Saito H, et al. Red-cell-volume distribution curves in diagnosis of glomerular and nonglomerular haematuria. Lancet 1988;1:908-11.

5. Shichiri M, Oowada A, Nishio Y, Tomita K, Shiigai T. Use of autoanalyser to examine urinary-red-cell morphology in the diagnosis of glomerular haematuria. Lancet 1986;2:781-2.

6. Racki S, Grzetić M, Prodan-Merlak Z, Vuksanović-Mikulicić S, SladojeMartinović B, Zivcić-Cosić S. Clinical use of phase-contrast microscopy in the differential diagnosis of microhematuria. Acta Med Croatica 2003; 57:11-6.

7. Ito CA, Pecoits-Filho R, Bail L, Wosiack MA, Afinovicz D, Hauser AB. Comparative analysis of two methodologies for the identification of urinary red blood cell casts. J Bras Nefrol 2011;33:402-7.

8. Chu-Su Y, Shukuya K, Yokoyama T, Lin WC, Chiang CK, Lin CW. Enhancing the detection of dysmorphic red blood cells and renal tubular epithelial cells with a modified urinalysis protocol. Sci Rep 2017;7:40521.

9. Fogazzi GB and Grignani S. Urine microscopic analysis-an art abandoned by nephrologists? Nephrol Dial Transplant 1998;13:2485-7.

10. Davis R, Jones JS, Barocas DA, Castle EP, Lang EK, Leveillee RJ, et al. Diagnosis, evaluation and follow-up of asymptomatic microhematuria (AMH) in adults: AUA guideline. J Urol 2012;188:2473-81.

11. Uno D, Kawakami S. UF-1000i/UF-500i clinical case study. 2nd ed. Kobe: Sysmex Corporation, 2014:1-35.

12. Jiang T, Chen P, Ouyang J, Zhang S, Cai D. Urine particles analysis: performance evaluation of Sysmex UF-1000i and comparison among urine flow cytometer, dipstick, and visual microscopic examination. Scand J Clin Lab Invest 2011;71:30-7.

13. Hyodo T, Kumano K, Sakai T. Differential diagnosis between glomerular and nonglomerular hematuria by automated urinary flow cytometer. Kitasato University Kidney Center criteria. Kitasato University Kidney Center criteria. Nephron 1999;82:312-23.

14. Nguyen GK. Urine cytology in renal glomerular disease and value of G1 cell in the diagnosis of glomerular bleeding. Diagn Cytopathol 2003;29: 67-73.

15. van der Zwet WC, Hessels J, Canbolat F, Deckers MM. Evaluation of the Sysmex UF-1000i ${ }^{\circledR}$ urine flow cytometer in the diagnostic work-up of suspected urinary tract infection in a Dutch general hospital. Clin Chem Lab Med 2010;48:1765-71.

16. Oyaert M and Delanghe J. Progress in Automated Urinalysis. Ann Lab Med 2019;39:15-22.

17. CLSI. Urinalysis; approved guideline. 3rd ed. CLSI GP16-A3. Wayne, PA: Clinical and Laboratory Standards Institute, 2009.

18. Broeren MA, Bahçeci S, Vader HL, Arents NL. Screening for urinary tract infection with the Sysmex UF-1000i urine flow cytometer. J Clin Microbiol 2011;49:1025-9.

19. Köhler H, Wandel E, Brunck B. Acanthocyturia-a characteristic marker for glomerular bleeding. Kidney Int 1991;40:115-20.

20. Levey AS, Stevens LA, Schmid CH, Zhang YL, Castro AF, 3rd, Feldman $\mathrm{HI}$, et al. A new equation to estimate glomerular filtration rate. Ann Intern Med 2009;150:604-12.

21. Pollock C, Liu PL, Györy AZ, Grigg R, Gallery ED, Caterson R, et al. Dysmorphism of urinary red blood cells-value in diagnosis. Kidney Int 1989; 36:1045-9.

22. Fuiano G, Mazza G, Comi N, Caglioti A, De Nicola L, lodice C, et al. Current indications for renal biopsy: a questionnaire-based survey. Am J Kidney Dis 2000;35:448-57.

23. Sultana T, Sultana T, Rahman MQ, Ahmed ANN. Evaluation of haematuria and use of phase contrast microscope: a short review. J Dhaka Med Coll 2011;20:63-7.

24. Santangelo L, Netti GS, Giordano P, Carbone V, Martino M, Torres DD, et al. Indications and results of renal biopsy in children: a 36-year experience. World J Pediatr 2018;14:127-33.

25. Jo Y-I. Diagnosis of primary glomerular diseases. Korean. J Med 2013; 84:6-12.

26. Albani JM, Ciaschini MW, Streem SB, Herts BR, Angermeier KW. The role of computerized tomographic urography in the initial evaluation of hematuria. J Urol 2007;177:644-8.

27. Crop MJ, de Rijke YB, Verhagen PC, Cransberg K, Zietse R. Diagnostic value of urinary dysmorphic erythrocytes in clinical practice. Nephron Clin Pract 2010;115:c203-12.

28. Stapleton FB. Morphology of urinary red blood cells: a simple guide in localizing the site of hematuria. Pediatr Clin North Am 1987;34:561-9. 